Short Paper

\title{
Die neue physiologische Störung "Grüne Beeren" bei der Sorte Gewürztraminer
}

\author{
The new physiological disorder "green berries" on the variety Gewürztraminer
}

La nuova malattia fisiologica "acini verdi" sulla varietà Gewürztraminer

\author{
Florian Haas ${ }^{1}$, Barbara Raifer ${ }^{1}$ \\ 1 Versuchszentrum Laimburg, Pfatten, Italien
}

\section{ABSTRACT}

The grape variety Gewürztraminer is known to be affected by two physiological disorders namely berry shrivel and bunch stem necrosis. During the season 2014 we noticed a new symptomatology type of ripening disorder on the variety. The new symptom showed not all berries fallowing the normal maturation stages, but single berries remaining at a soft but green stage till harvest. This work shows the results of first attempts to find causes for the symptom as well as the resulting approach to mitigate symptoms. Applications of magnesium leaf fertilizer showed first promising results against this putative disorder reducing incidence and severity of the symptom.

\section{KEYWORDS}

Ripening disorder, Magnesium, green berries, Gewürztraminer,

\section{CITE ARTICLE AS}

Haas Florian, Raifer Barbara (2021). The new physiological disorder "green berries" on the variety Gewürztraminer.

Laimburg Journal 03/2021 DOI: $10.23796 / \mathrm{LJ} / 2021.011$

\section{CORRESPONDING AUTHOR}

Florian Haas

Laimburg 6, Pfatten, 39040 Auer (BZ), Italien

florian.haas@laimburg.it +390471969613 


\section{EINLEITUNG}

\section{BESCHREIBUNG DER NEUEN PHYSI- OLOGISCHEN STÖRUNG}

Seit dem Jahr 2000 ist die physiologische Störung Traubenwelke oder "berry shrivel" bei der Sorte Gewürztraminer in Südtirol bekannt [1]. Ebenso kann die Störung Stiellähme bei der Sorte gefunden werden [2]. Diese bereits bekannten Störungen des $\mathrm{Ge}$ würztraminer führten daher schon zu großen Herausforderungen in Bezug auf dessen regelmäßige und vollständige Reife der Beeren, welche die Grundlage für die Produktion von hochqualitativem Gewürztraminer Wein darstellt [3].

Nach dem sehr feuchten Sommer 2014 trat im Laufe der Reifephase eine neue Art der Reifestörung bei Gewürztraminer auf. Hierbei fiel den Winzern auf, dass mehr oder weniger viele Beeren nicht den normalen Reifeprozess durchliefen, sondern grün blieben. Diese Beobachtung wurde 2014 vor allem im Eisacktal gemacht und wurde zu einem goßen Problem für die betroffenen Traubenproduzenten. Im Jahr 2016 waren dann in vielen Anlagen der Sorte Gewürztraminer in ganz Südtirol "Grüne Beeren" anzutreffen, so auch in Tramin, wo eine beachtliche Fläche mit dieser Sorte angebaut wird. Die grünen Beeren, die es zu entfernen galt, da sie sauer, geschmacklos und zum Teil bitter waren, brachten bei der Lese einen nicht zu unterschätzenden Mehraufwand für die Winzer mit sich. Aus diesem Grund wurde das Auftreten 2016 intensiv verfolgt und dokumentiert, vor allem um zu verstehen, ob es sich hierbei um eine abgeänderte Form der Traubenwelke oder um ein neues Pathogen handelt.

\section{SYMPTOMATIK}

Bereits bald nach Reifebeginn zeichnete sich im Jahr 2016 die uneinheitliche Reife einzelner Beeren ab, die Beeren blieben grün und ließen keinen Farbumschlag erkennen. Diese Beeren zeigten zunächst keine weiteren Veränderungen, blieben prall und wiesen am Stielgerüst keine Schädigung auf. Erst etwa drei Wochen nach dem fehlenden Farbumschlag wurden an den Stielen der betroffenen Beeren, nicht immer aber häufig, mehr oder weniger ausgeprägte Nekrosen sichtbar, wie sie für Stiellähme typisch sind (Abb. 1, Abb. 2, Abb. 3, Abb. 4). In weniger stark befallenen Anlagen waren an der sonnenexponierten Seite der Trauben oftmals annähernd keine "Grünen Beeren" zu sehen.

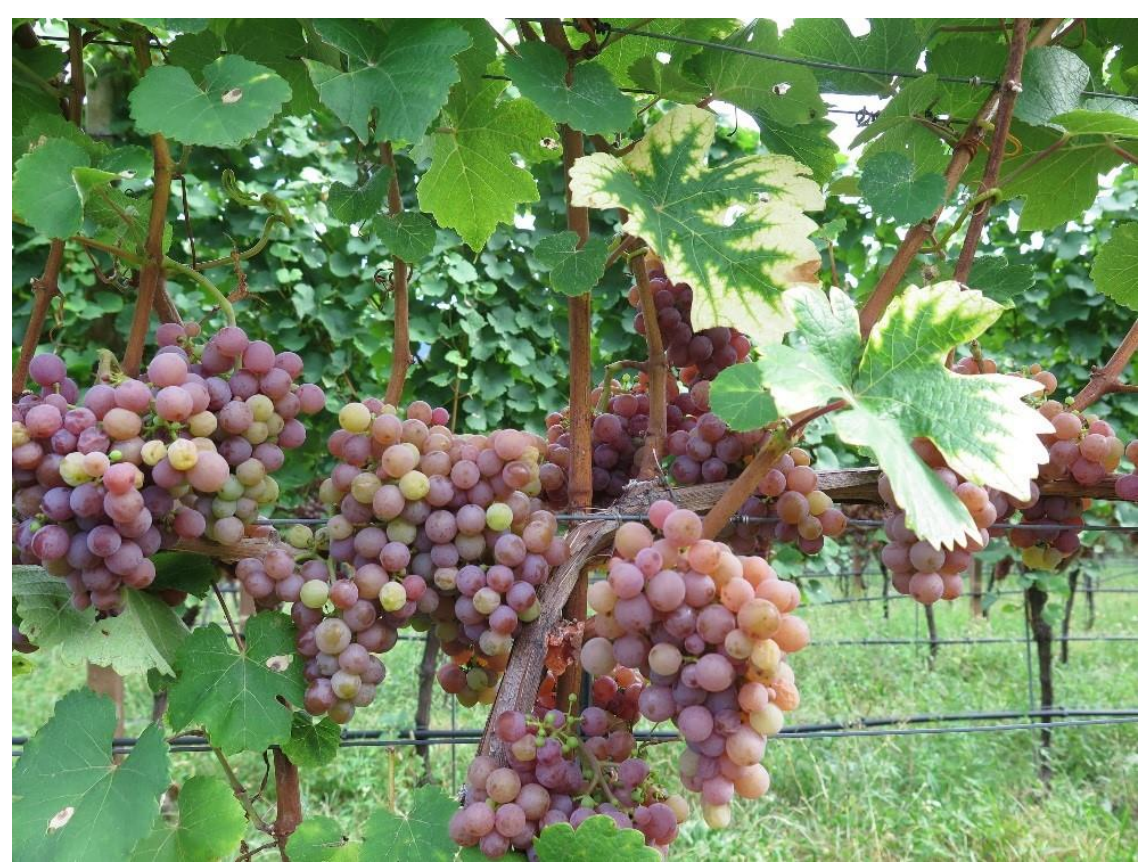

Abb. 1: "Grüne Beeren" bei Gewürztraminer kamen 2016 häufig zusammen mit deutlichen Mangnesiummangel-Symptomen an den Blättern vor. // „Green berries“ on Gewürztraminer appeared 2016 often with obvious magnesia deficit symptoms.
Ihr Auftreten beschränkte sich auf die weniger gut exponierte Seite oder auf das Innere der Trauben. Im Laufe der Reifeentwicklung schrumpften die befallenen Beeren mehr oder weniger und nahmen gegen Reife der Trauben oft eine gelbliche Farbe an. Die Beeren blieben aber sauer und geschmacklos.
Auffallend war 2016, dass viele betroffene Gewürztraminer-Anlagen mehr oder weniger ausgeprägte Magnesiummangel-Symptome an den Blättern zeigten. "Grüne Beeren" traten zum Teil aber auch in Anlagen ohne Blattveränderungen oder mit unspezifischen, leichten Blattaufhellungen auf. Hingegen waren in den Anlagen mit deutlichen Magnesiummangel-Symptomen durchwegs

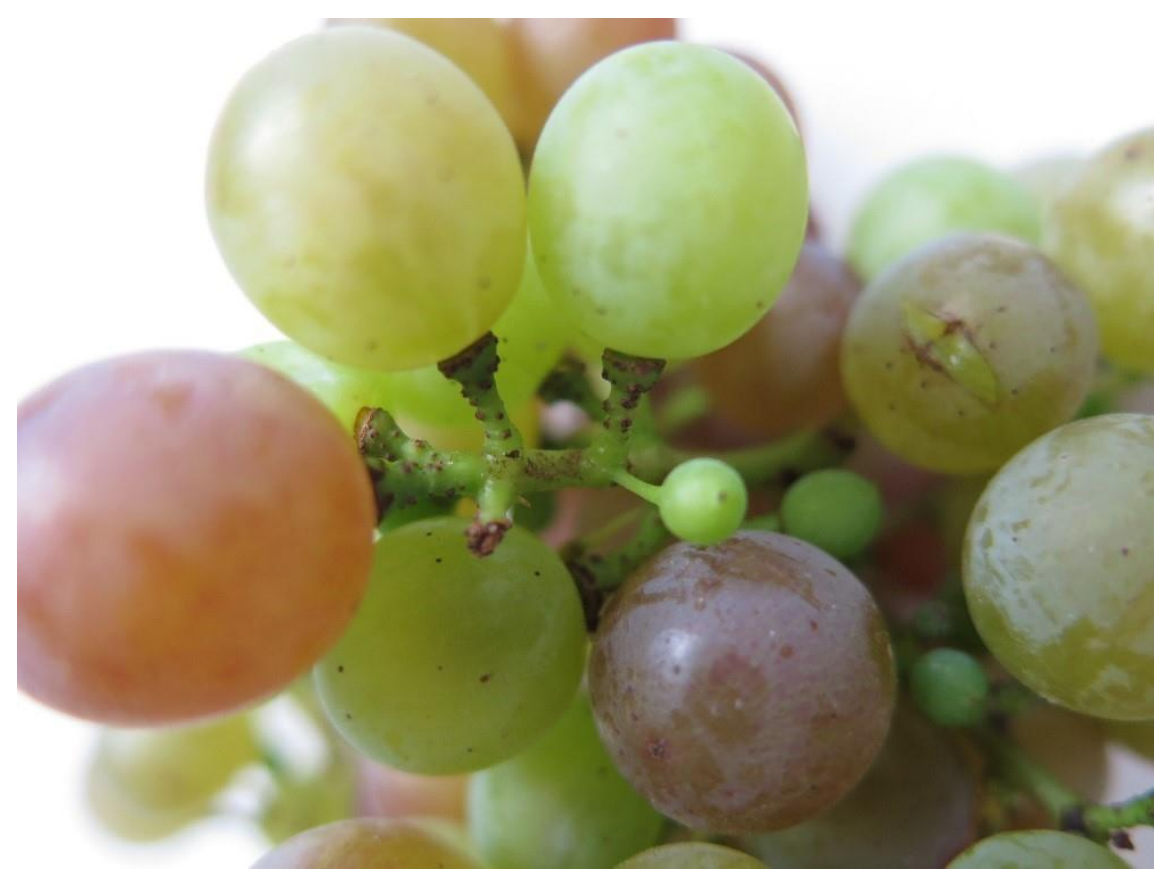

Abb. 2: Das Stielgerüst der betroffenen Beeren zeigte zunächst keine Auffälligkeiten. // The rachis of affected berries did not show any distinctive feature. 


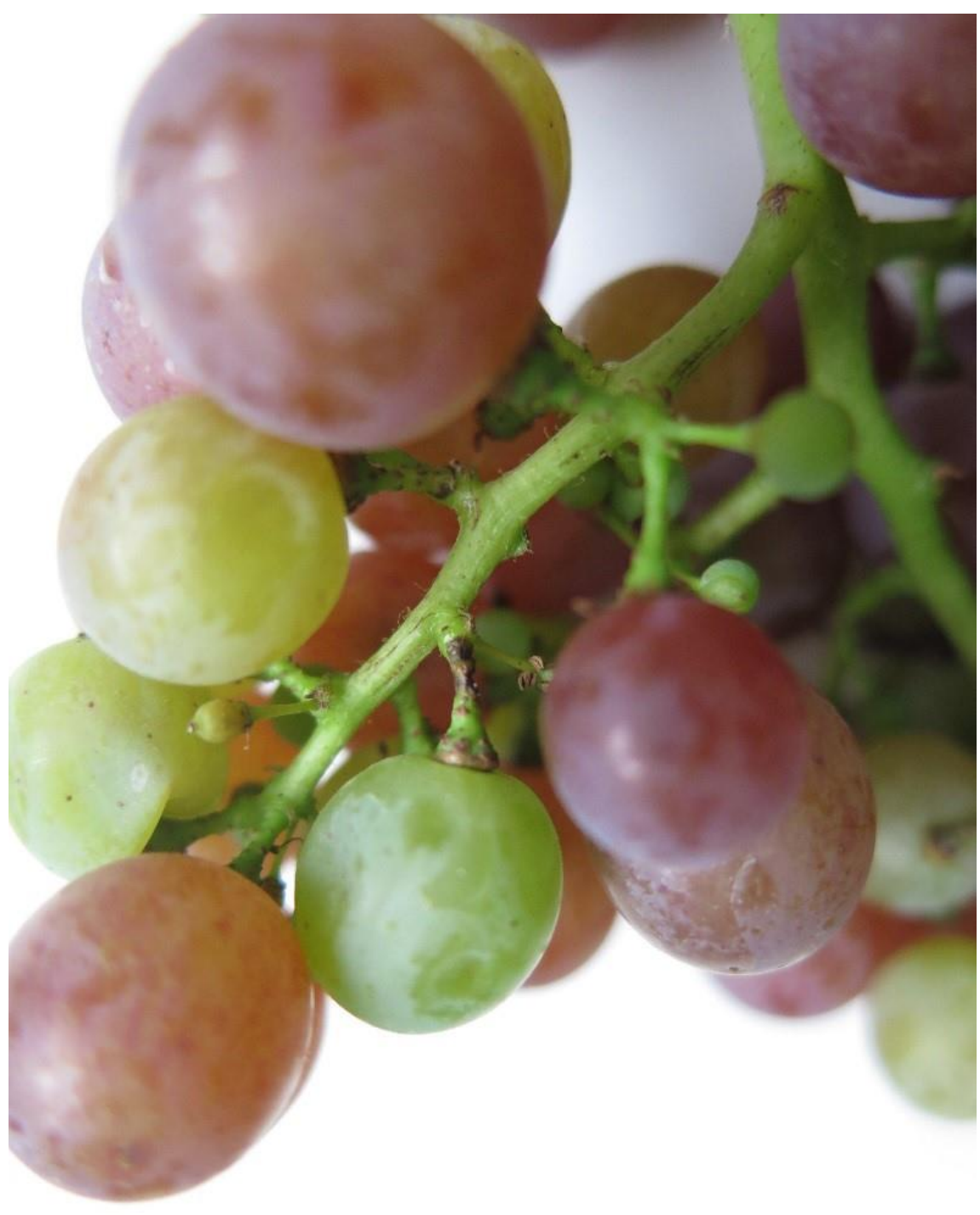

Abb. 3: Erst etwa drei Wochen nach dem Sichtbarwerden der "Grünen Beeren" traten an vielen Beerenstielen betroffener Beeren kleine dunkle Einschnürungen auf. // Only after three weeks after appearance of the "green berries" dark constriction rings emerged on their pedicels.

auch "Grüne Beeren" zu finden. In den nachfolgenden Jahren waren nicht so ausgeprägte Magnesiummangel-Symptome zu finden wie 2016, ohne dass zugleich auch "Grüne Beeren" aufgetreten wären.

Vom Erscheinungsbild her kann das Phänomen einerseits als eine auf einzelne Beeren beschränkte Traubenwelke bezeichnet werden, wobei klassische Traubenwelke bei Gewürztraminer in früheren Jahren meistens ganze Traubenteile bis hin zur gesamten Traube betroffen hat [4] [5]. Andererseits besteht auch eine Nähe zu Stiellähme, da die Stiele der "Grüne Beeren" im Laufe der Reifephase oft deutliche StiellähmeSymptome entwickelten.
Befall an "Grünen Beeren" über die gesamte Vegetationsperiode eine ungenügende Magnesiumversorgung aufwiesen. Einige Parzellen mit ebenfalls mangelhafter Magnesiumversorgung zeigten allerdings kaum oder nur wenig "Grüne Beeren". Dies könnte auf gezielte Magnesiumbehandlungen der Trauben dieser Anlagen zurückzuführen sein.

Auch in einem weiteren Versuch bei der Sorte Gewürztraminer in Tramin traten 2016 in zwei von sechs beobachteten Gewürztraminer-Anlagen "Grüne Beeren" auf: In einer Anlage nur wenige, in der zweiten sehr viele. In beiden Anlagen waren die Magnesiumgehalte der Böden deutlich niedriger als die der befallsfreien Anlagen, laut den Blattanalysen waren die Magnesiumwerte besonders der stark betroffenen Anlage im Mangelbereich.

Im Falle der "Grünen Beeren" im Jahr 2016 scheint somit eine eher niedrige Magnesiumversorgung der Böden bzw. der Reben laut Blattanalysen dazu beigetragen zu haben, dass zeitweise zu wenig Magnesium für die gleichmäßige Versorgung der Beeren zur Verfügung stand. Analysen der Blattstiele, die besser als die Blattspreiten widerspiegeln was die Reben gerade aufnehmen, zeigten bis zu dreimal so hohe Magnesiumwerte in Anlagen mit gut versorgten Böden gegenüber den weniger gut versorgten.

Im Gegenteil hierzu wurden in vergangenen Versuchen zu Traubenwelke bei Gewürztraminer in den Varianten mit intensivem Welkeauftreten niedrige Kaliumwerte im Blatt und in der Beere gefunden bei zugleich erhöhten Magnesiumgehalten. Hierbei unterscheidet sich somit das Symptom der "Grünen Beeren" und jenes der Traubenwelke deutlich [1].

Es ist möglich, dass noch weitere Faktoren hierbei eine Rolle spielen, die aber derzeit nicht eindeutig zugeordnet werden können. So ergab sich im Jahr 2016 ein Verdachtsmoment bezüglich der Anzahl und des Zeitpunktes der Kalium-Phosphit-Behandlungen. Außerdem schien rein optisch die Wuchsstärke eine Rolle zu spielen. Laut dem Nmin-Gehalt im Boden, den Blatt- und Mostanalysen ergab sich aber kein Zusammenhang zwischen dem Auftreten der "Grünen Beeren" und der Stickstoffverfügbarkeit und somit der Wüchsigkeit der Anlage. 


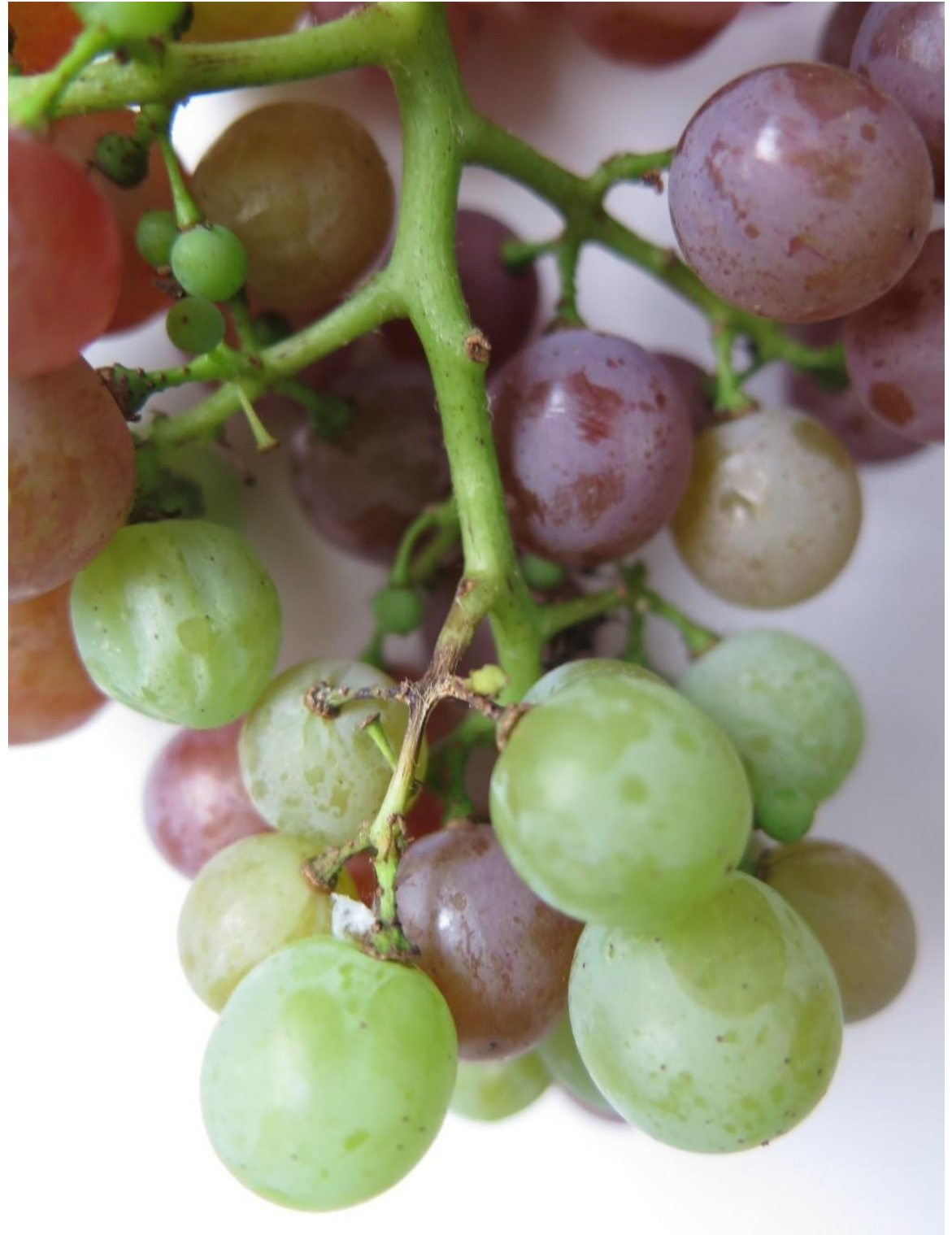

Abb. 4: Diese entwickelten sich dann zum Teil zu klassischen Stiellähme-Symptomen. // Some of them seemed most likely to develop as the classic stem rot necrosis symptoms.

\section{MAGNESIUM WICHTIG FÜR DIE REIFE}

Da Magnesium im Phloem transportiert werden kann, wird es bei ungenügender Verfügbarkeit und Aufnahme aus dem Boden aus den älteren Blättern wieder ausgelagert. In der Folge entstehen die typischen Mangelsymptome an den Blättern der Traubenzone. Bereits bevor es aber zu diesen offensichtlichen Mangelsymptomen kommt, wirkt sich ein Magnesiumdefizit hemmend auf die Verlagerung von Zucker und Aminosäuren aus den Blättern in die Früchte und in andere Organe der Rebe aus [6]. Dabei ist immer zu berücksichtigen, dass es sich bei physiologischen Störungen wie der Stiellähme und wohl auch bei den "Grünen
Beeren" nicht unbedingt um ein absolutes Magnesiumdefizit in den Böden oder in den Organen der Rebe handelt. Vielmehr dürfte durch das Zusammenwirken ungünstiger Faktoren zeitweilig der Magnesiumbedarf nicht gedeckt werden können. 2016 führten die anhaltenden Niederschläge im Frühsommer generell zu deutlich höherer Kaliumaufnahme als im Vorjahr, da es schnell und leicht von den gefallenen Regenmengen gelöst werden kann. Kalium hemmt bekanntlich die Magnesiumaufnahme [7] und kann somit zu einem Magnesiummangel in den Rebpflanzen führen. Da Magnesium auch für die Entwicklung des Neuzuwachses gebraucht wird, wie auch für den Proteinaufbau und die Aktivierung vieler Enzyme, ist es zudem verständlich, dass umso mehr
Magnesium erforderlich ist, je stärker das vegetative Wachstum ist und je länger dieses anhält.

\section{BLATTDÜNGEVERSUCH}

Aufgrund dieser Beobachtungen und Erfahrungen wurde ein Blattdüngeversuch durchgeführt, um abzuklären ob der Magnesiummangel definitiv ein Auslösefaktor für diese neue physiologische Störung sein kann und ob eine gute Magnesiumversorgung das Auftreten diese Symptomatik lindern kann. Aus einem Versuch zur Traubenwelke von [8] ist bekannt, dass das Magnesium Chlorid $(\mathrm{MgCl} 2)$ besser in die Pflanze aufgenommen werden konnte als das klassische Magnesiumsulfat (MgSO4). Daher kamen im vorliegenden Versuch beide Formulierungen zum Einsatz.

\section{METHODEN}

\section{VERSUCHSDURCHFÜHRUNG}

Im Jahr 2016 wurden 30 Anlagen ausgewählt, welche im Jahr 2014 schon diese Symptomatik aufwiesen. In diesen Anlagen wurden jeweils bei der Blüte und bei Reifebeginn, wie später erklärt, Nährstoffanalysen der Blätter durchgeführt, um einen Überblick über die generelle Nährstoffversorgung der Anlagen zu erhalten.

Um den Blattdüngeversuch durchzuführen, wurde in den Jahren 2018 und 2019 eine Anlage in Tramin Söll $\left(46^{\circ} 21^{\prime} 24.4^{\prime \prime} \mathrm{N}\right.$ $11^{\circ} 14^{\prime} 28.1^{\prime \prime E}$ ) ausgewählt, welche 2016 ein intensives Auftreten der Symptomatik gezeigt hat. Im Jahr 2020 wurde der Versuch in eine Gewürztraminer-Anlage in Klausen (46 38'52.2"N 11³4'41.7"E) verlegt. Für die Applikation der Magnesiumblattdünger wurden zwei verschiedene Formulierungen ausgewählt: Magnesiumchlorid (MgCL2) und Magnesiumsulfat (MgSO4). Die Blattdünger wurden mittels einer 15 L-Rückenspritze appliziert. Die verwendete Wassermenge entpricht der klassischen Pflanzenschutzapplikation, welcher in Südtirol Blattdünger wie Magnesium beigemengt und mitappliziert werden. Somit entspricht auch die Applikation im Versuch jener von $10 \mathrm{hl} / \mathrm{ha}$ und einer Konzentration von $500 \mathrm{~g} \mathrm{Mg} / \mathrm{hl}$. Um die Aufnahmefähigkeit des Blattdüngers in den Pflanzen zu optimieren, wurde der Brühe noch etwas Zitronensäure beigemengt, um den $\mathrm{pH}$-Wert einzustellen. Die Blattdünger wurden in dreifacher Wiederholung mit drei randomisierten Blöcken der unbehandelten Kontrolle 


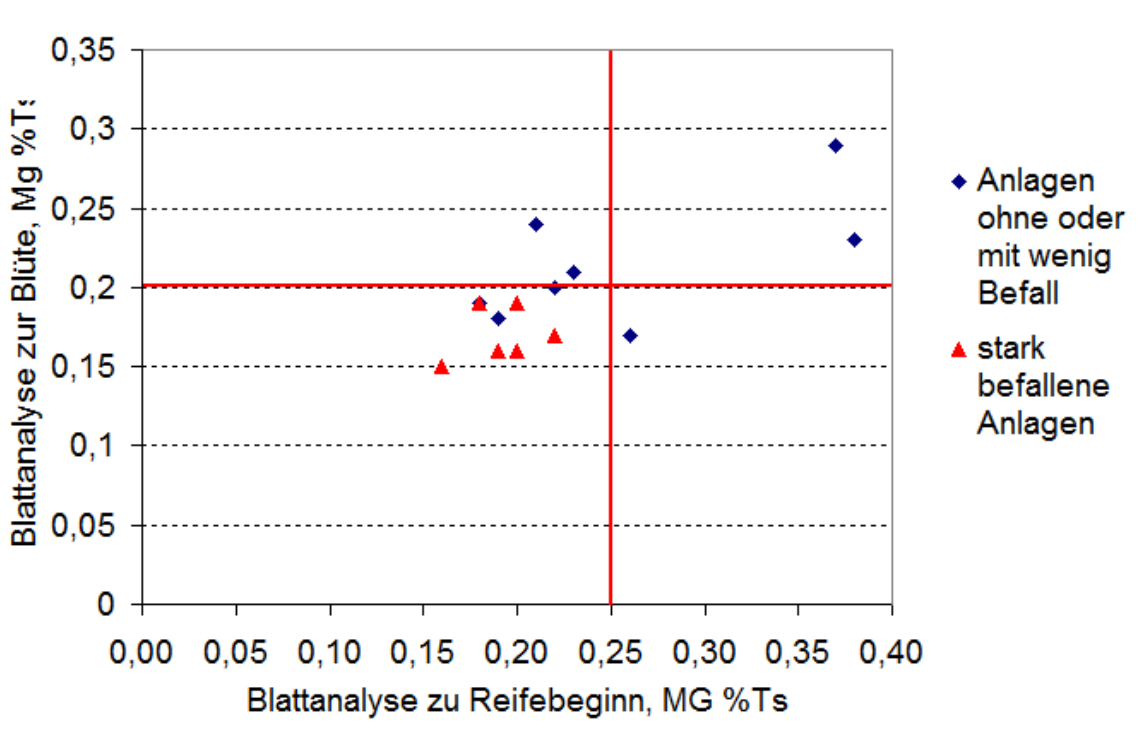

Abb. 5: Magnesiumversorgung der Kontrollanlagen bei Gewürztraminer im Eisacktal 2016; die roten Linien geben jeweils den Schwellenwert zum normalversorgten Bereich an. // Magnesia content in the control vineyards on Gewürztraminer in the Eisacktal valley in 2016; red lines mark the threshold of the sufficient range for Magnesia-content.

gegenübergestellt. Die Wiederholungen beinhalteten jeweils 15 Reben und die Behandlungsreihe war durch eine unbehandelte Reihe von der nächsten Behandlungsreihe getrennt, um Abdriftsproblemen vorzubeugen. Die Applikationen wurden jeweils am Abend nach Sonnenuntergang und wenn möglich bei bewölkter Witterung durchgeführt, um eine möglichst lange Aufnahmedauer mit offenen Spaltöffnungen der Blätter und Trauben zu gewährleisten. In den Jahren 2017 und 2018 wurden die Magnesiumapplikationen dreimal von Anfang Juli (phänologisches Stadium: Trauben schließen) bis Mitte August (phänologisches Stadium: Reifebeginn) durchgeführt, während 2019 und 2020 noch ein früherer Termin Tab. 1: Auswertung der Symptomatik "grüne Beeren" in der Anlage Gewürztraminer in Klausen am 22.09.2020 // Evaluation of "green berry" symptoms in the experimental vineyard in Klausen at 22.09.2020.

\section{Befallsklasse Anteil an "grünen Beeren"}

incidence class Percentage of "green berry"

$\begin{array}{cc}0 & \text { kein Befall } \\ 1 & 0-3 \% \\ 2 & 3-6 \% \\ 3 & 10-25 \% \\ 4 & 25-50 \% \\ 5 & 50-90 \% \\ 6 & 90-100 \%\end{array}$

Ende Juni (phänologisches Stadium: Schrotkorn) hinzugefügt wurde, um die Aufnahmefähigkeit der noch vegetativen Beeren zu nützen und somit die Reben schon frühzeitig sowohl am Blatt als auch am Geschein mit Magnesium zu versorgen.

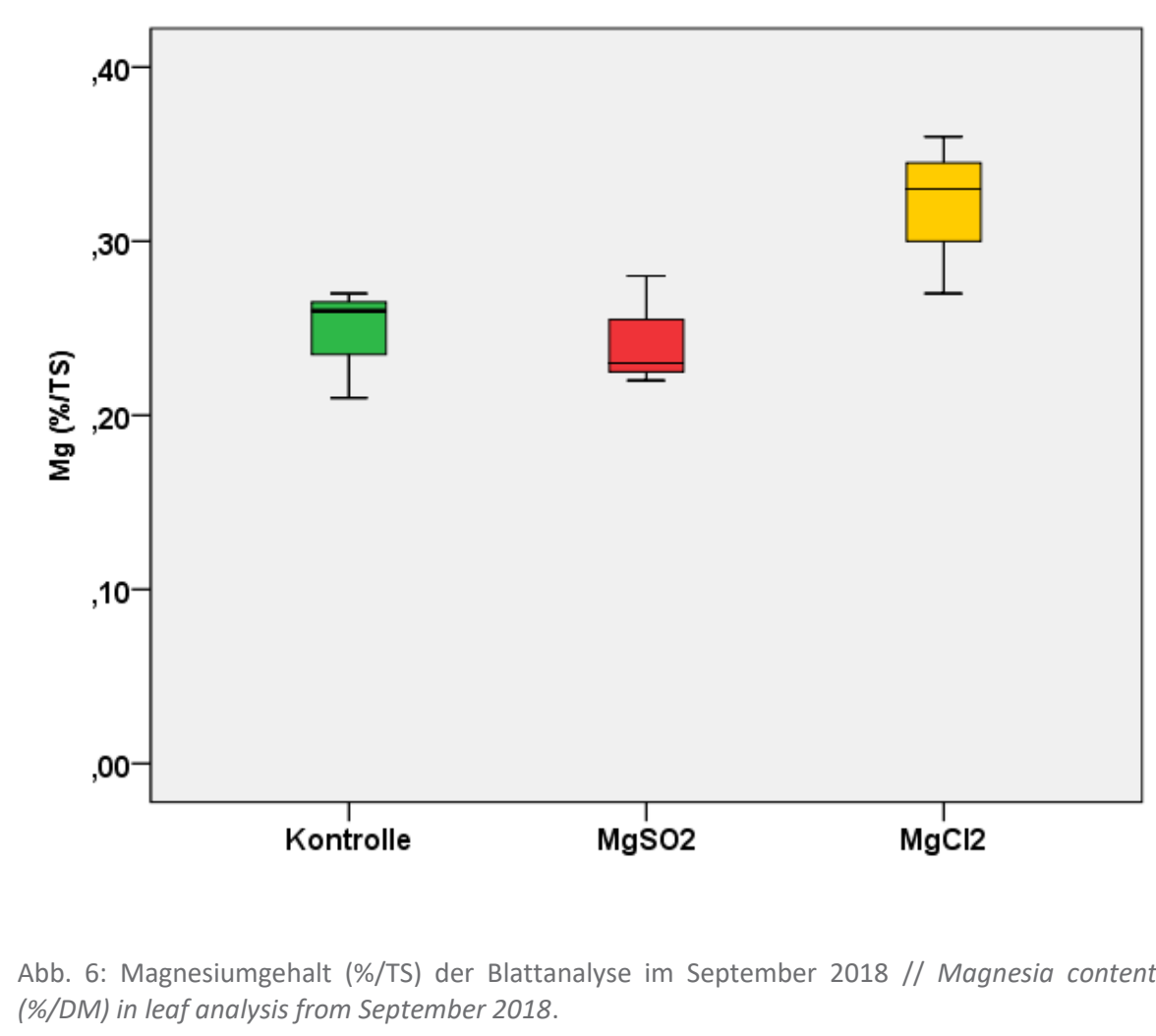

Abb. 6: Magnesiumgehalt (\%/TS) der Blattanalyse im September 2018 // Magnesia content (\%/DM) in leaf analysis from September 2018.
Um die Nährstoffgrundversorgung der Anlagen bewerten zu können, wurde eine repräsentative Bodenanalyse der Versuchsanlagen nach dem VDLUFA Methodenbuch im Bodenlabor des Versuchszentrums Laimburg durchgeführt.

\section{AUSWERTUNGS- UND ANALYSE- METHODEN}

Um die Aufnahme der Blattdünger zu überprüfen, wurden Blattanalysen in unterschiedlichen Entwicklungsstadien der Rebe aus einer Stichprobe von 20 repräsentativen Blättern aus jeder Wiederholung durchgeführt. Auch Nährstoffanalysen der Beeren (75 Beeren/Wiederholung) sollten Aufschluss über die Aufnahme und Verteilung des Magnesiums bis in die Beere liefern. Die Beeren der Trauben wurden kurz vor der Ernte mittels einer spectroskopischen Analysemethode (FTIR WineScan ${ }^{\text {TM }}$, FOSS, Denmark) auch auf ihre qualitätsgebenden Eigenschaften wie Zucker ( ${ }^{\circ} \mathrm{KMW}$ ), Säuren (titrierbare Gesamtsäure, Weinsäure $(\mathrm{g} / \mathrm{L})$ und Apfelsäure (g/L)), ph-Wert und hefeverwertbarer Stickstoff-Gehalt (HVS in $\mathrm{mg} / \mathrm{L}$ ) untersucht. Die Auswertung der Befallshäufigkeit und Befallsstärke wurde anhand 5 Befallsklassen, wie in Tabelle 1 abgebildet, durchgeführt. Dabei wurden 100 Trauben je Wiederholung berücksichtigt. 


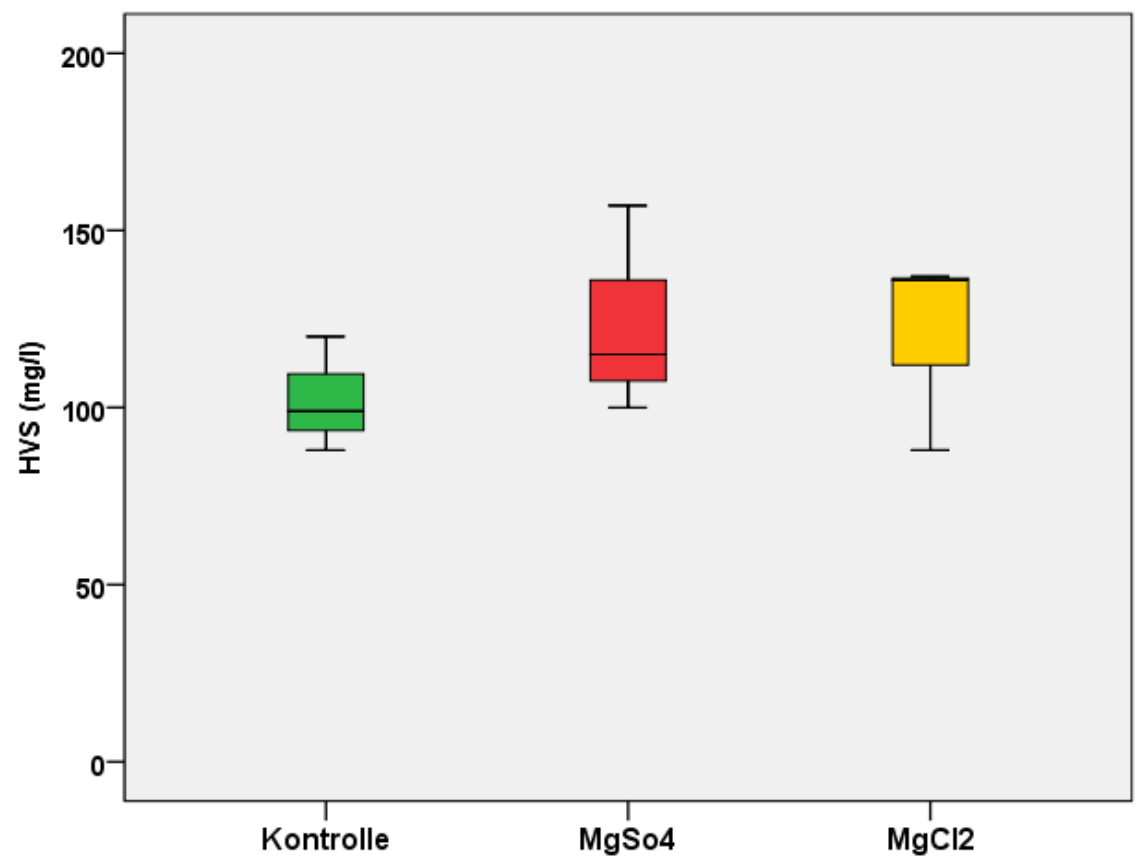

Abb. 7: Gehalt an hefeverwertbarem Stickstoff $(\mathrm{mg} / \mathrm{l})$ in der Beere // Content of yeast assimilable nitrogen ( $\mathrm{mg} / \mathrm{l})$ in berries.

\section{ERGEBNISSE}

Die Bodenanalyse der Anlage in Tramin ergab einen schluffigen Lehm, welcher recht gut mit Humus versorgt war. Der pH-Wert war mit 7,4 etwas hoch, doch typisch für die

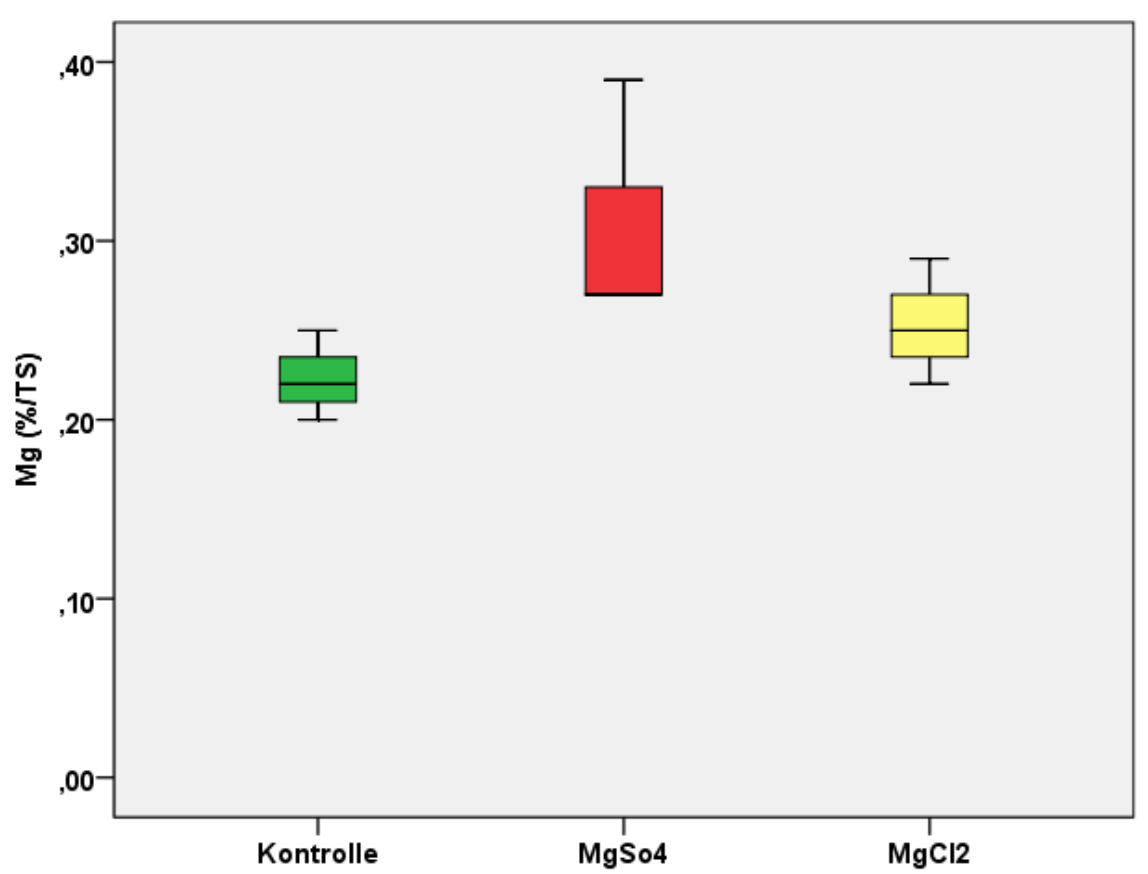

Abb. 8: Magnesiumgehalt der Blätter (\%/TS) // Magnesia content in leafs (\%/DM).
$17 \mathrm{mg} / 100 \mathrm{~g}$ im Oberboden und 13 und 16 mg/100 g im Unterboden. In Klausen waren diese beide Nährstoffe mit 24 und $16 \mathrm{mg} / 100 \mathrm{~g}$ im Oberboden und 13 und $12 \mathrm{mg} / 100 \mathrm{~g}$ im Unterboden zu finden. Somit waren im Oberboden der Anlage in Klausen deutlich mehr Kalium zu finden als in Tramin.

Die Anlage in Tramin verfügt über keine Bewässerungsanlage, da es sich um einen sehr tiefgründigen Boden handelt. Die Anlage in Klausen hingegen, muss aufgrund ihrer Steilheit und ihres relativ flachen Bodens bewässert werden.

Aus den Blattanalysen der 30 Anlagen aus dem Jahr 2016 im Eisacktal konnte ein klarer Zusammenhang zwischen dem Magnesiumgehalt der Reben und dem Auftreten der Symptomatik festgestellt werden. In der Abbildung 5 sind die Magnesiumgehalte der Blätter bei der Blüte dem Magnesiumgehalt bei Reifebeginn gegenübergestellt. Es fällt auf, dass nicht alle Anlagen ohne "Grüne Beeren" sehr gut mit Magnesium versorgt sind, jedoch geht klar hervor, dass alle Anlagen, welche "Grüne Beeren" aufwiesen, während der gesamten Vegetationsphase einen zu niedrigen Gehalt an Magnesium hatten.

Die Blattanalysen des Magnesium-Blattdüngeversuchs in Tramin zeigten im September 2018 eine deutlich bessere Aufnahme des Magnesiumchlorids gegenüber der Sulfatformulierung, welche sich nicht von der Kontrollvariante unterscheiden konnte (Abb. 6).

Im selben Jahr wurden die Trauben auf ihre qualitätsgebenden Inhaltsstoffe untersucht. Bei diesem sogenannten Reifetest konnten keine Veränderungen im Zucker- oder Säuregehalt, beziehungsweise des $\mathrm{pH}$-Wertes festgestellt werden. Einzig der Gehalt an hefeverwertbarem Stickstoff (HVS) konnte tendenziell gesteigert werden (Abb. 7).

Die Nährstoffanalyse der Beeren kurz vor der Lese hat 2019 leider keine Unterschiede zwischen den Versuchsvarianten feststellen lassen.

In den Jahren 2018 und 2019 konnte keine Auswertung bezüglich des Effektes der Blattdüngerbehandlungen auf das Auftreten der "Grünen Beeren" durchgeführt werden, da in der Versuchsanlage keine Symptome dieser physiologischen Störung aufgetreten sind. Aus diesem Grund wurde 2020 der Blattdüngeversuch in Klausen in einer anderen privaten Versuchsanlage weitergeführt. 


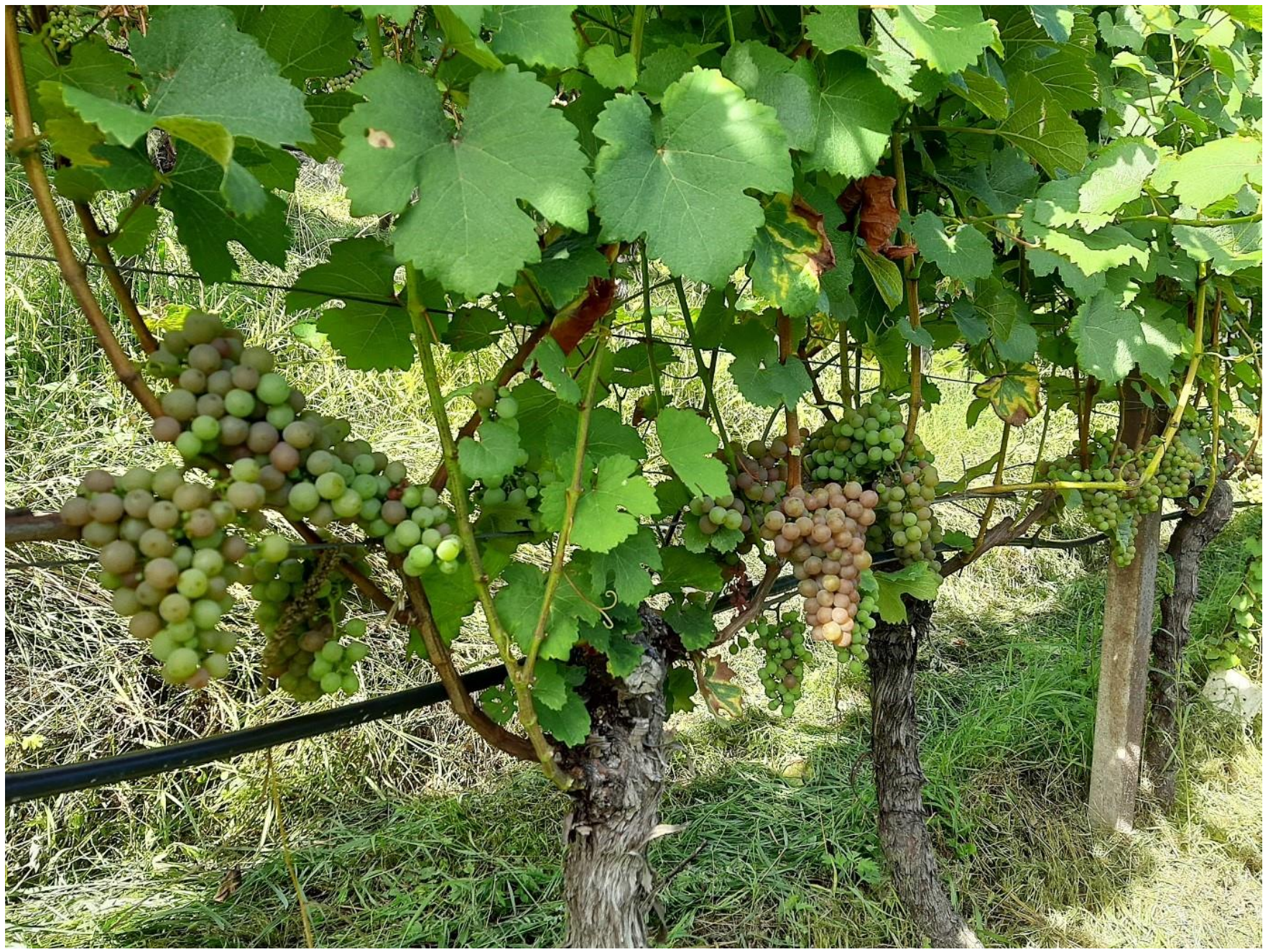

Abb. 9: Unregelmäßiger Reifebeginn bei Gewürztraminer in Klausen am 19.08.2020 // Irregular veraison on Gewürztraminer in Klausen at 19.08.2020.

Die Blattanalysen wurden bei Reifebeginn am 19.08.2020 durchgeführt. Die Ergebnisse dieser Analyse zeigten eine bessere Versorgung mit Magnesium der Blätter der mit Blattdünger behandelten Varianten (Abb. 8). Das Magnesiumsulfat wurde in dieser Anlage leicht besser aufgenommen als das Chlorid.

Schon während des Reifebeginns sind 2020 einige Trauben mit sehr unregelmäßigem Farbumschlag aufgefallen (Abb. 9).

Um jedoch sicher zu gehen, dass es keine Nachfärbung gibt, wurde die Symptomauswertung erst kurz vor der Lese am 22.09.2020 durchgeführt. In den vergangenen Jahren konnte beobachtet werden, dass es manchmal später nach dem Reifebeginn noch einige Beeren schafften nachzureifen. Repräsentative Beispielbilder für die Aus- wertung der Befallsstärke sind in der Abbildung 10 abgebildet.

Die Ergebnisse der Auswertung der Befallshäufigkeit konnten hierbei sehr klare Ergebnisse liefern. Es konnte eine signifikante Verringerung des Auftretens von Trauben mit "Grüne Beeren"-Symptome erkannt werden $(p=0,000)$ (Abb. 11). Die mit MagnesiumBlattdünger behandelte Variante konnte 2020 über 50\% mehr Trauben ohne Symptome aufweisen als jene der Kontrollvariante. Dennoch war auch in den behandelten Varianten die Häufigkeit der Trauben mit Symptomen bei MgSO 4 mit 54,04\% und bei $\mathrm{MgCl} 2$ mit 56,99\% sehr hoch. Zwischen den beiden Formulierungen konnte hierbei kein Unterschied festgestellt werden.

In der Abbildung 12 wird der Anteil an befallenen Trauben bezüglich ihrer Befallsstärke dargestellt. Es wird dabei deutlich, dass im
Jahr 2020 und in dieser Anlage die beiden Formulierungen keine nennenswerten Unterschiede hervorbrachten, auch wenn die Variante MgSO4 ganz leicht bessere Werte ergab. Auffällig ist auch, dass in der Kontrollvariante der Anteil an stark befallenen Trauben, also Trauben der Befallsklasse 4 mit 25 $50 \%$ "Grünen Beeren" und Befallsklasse 5 mit 50-90\% "Grünen Beeren", im Verhältnis zu den behandelten Varianten signifikant höher ist $(p=0,000)$.

Dieses Ergebnis bestärkt den Einsatz von Magnesium-Blattdünger zur Verringerung der "Grüne Beeren"-Symptomatik. Die Befallshäufigkeit von immerhin über $50 \%$ der Trauben war 2020 sicherlich sehr hoch, jedoch konnte die Befallsintensität im Feld in den mit Magnesium-Blattdüngern behandelten Varianten, als deutlich geringer erkannt werden. 

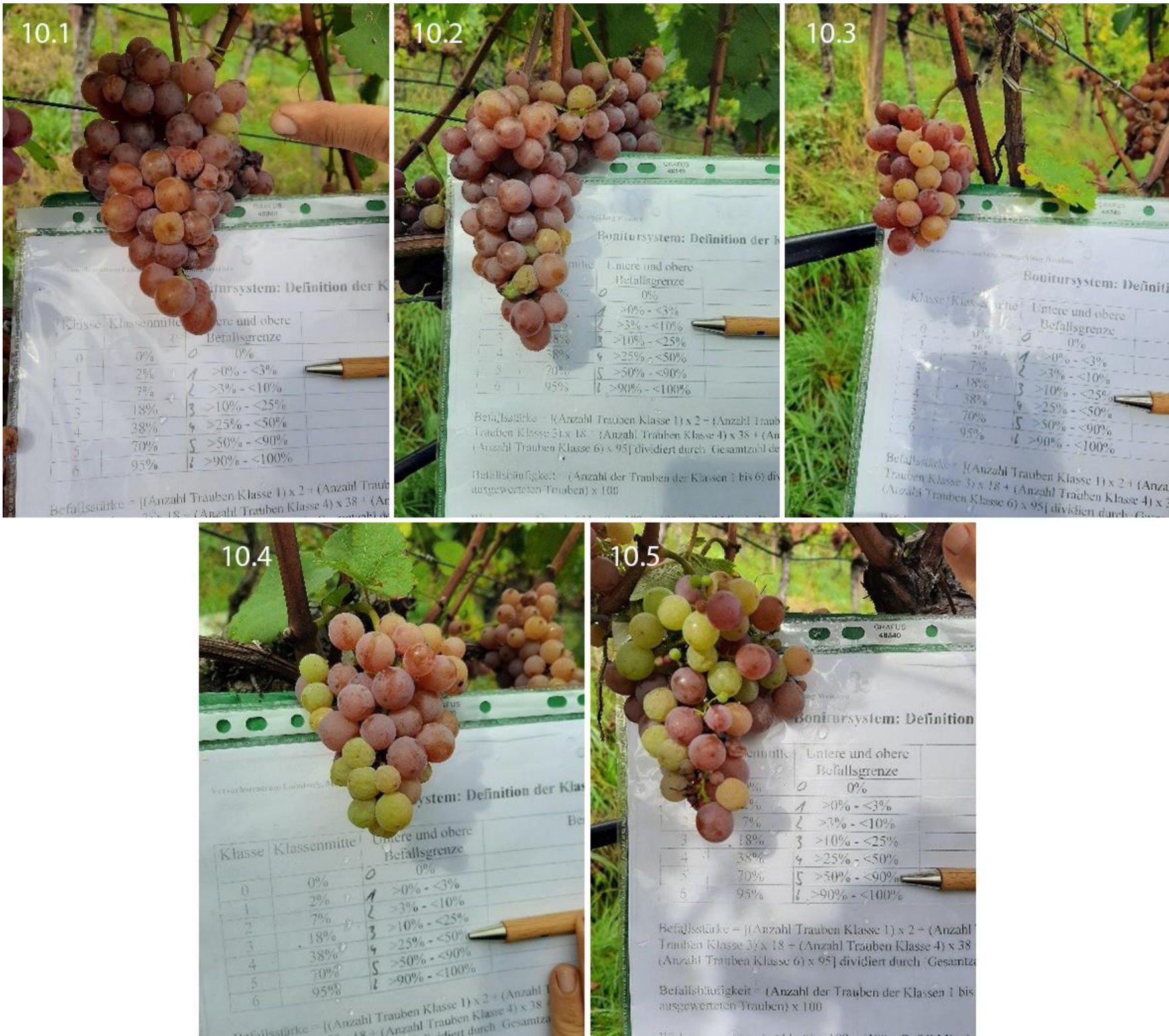

Abb. 10: Repräsentative Trauben der Befalls Klassen 1-5 wie in Tabelle 1 wiedergegeben // Grapes of representative clusters for the affection classes 1-5 as indicated in table 1.

\section{ZUSAMMENFASSUNG}

Die physiologische Störung "Grüne Beeren", welche oftmals sehr an die Symptome der Traubenwelke erinnert, ist ein sehr komplexes Thema. Nach diesen ersten Jahren der Ursachenforschung und Entwicklung einer Bekämpfungsstrategie, konnten schon einige Fortschritte erzielt werden. Der Ursachenkomplex ist noch nicht vollständig aufgeklärt, doch ist der Magnesiumgehalt und das Magnesium-Kalium-Verhältnis sicherlich entscheidend für das Auftreten dieser Symptomatik. Leider hat die Jahrgangswitterung einen sehr starken Einfluss auf die Ausprägung der Symptome, da sie maßgeblich die Nährstoffaufnahme bestimmt. Somit ist es sehr schwierig regelmäßig auswertbare Symptome in den Versuchsanlagen vorzufinden.

Die bisherigen Praxisberichte und die Versuchserfahrungen des Jahres 2020 erlauben es jedoch, unsere Hypothesen zu bestärken und in Anlagen mit "Grünen Beeren" zwei bis vier Bittersalz-(MgSO4)-Behandlungen vor Reifebeginn anzuraten. Die bisherigen Ergebnisse konnten bis dato noch keine klare Unterscheidung zwischen den beiden Magnesiumformulierungen hervorbringen, weder in Bezug auf die Aufnahme in die Pflanze noch gegenüber dem Auftreten der Symptome.
Aus diesem Grund führt die Arbeitsgruppe Physiologie und Anbautechnik bereits einen weiteren Versuch durch, um die Wirksamkeit der Magnesium-Blattdünger zu bestätigen und deren optimalen Einsatzzeitpunkt abzuklären. 


\section{Befallshäufigkeit Grüne Beeren GT Klausen 2020}

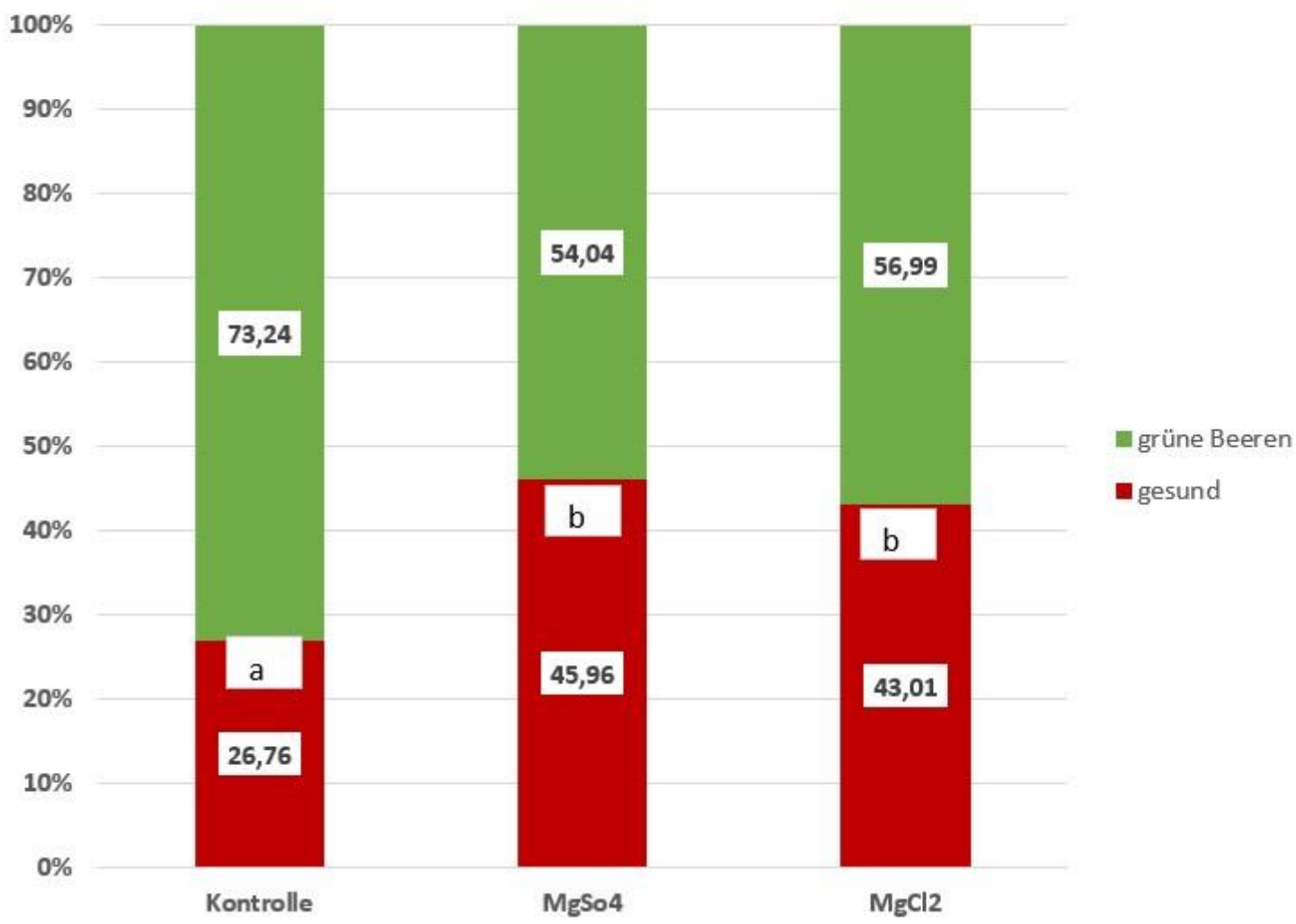

Abb. 11: Befallshäufigkeit von Trauben mit "Grünen Beeren" zwischen den Versuchsvarianten // Grape affection frequency with "green berries" among the thesis'.

Befallsstärke Grüne Beeren GT Klausen 2020

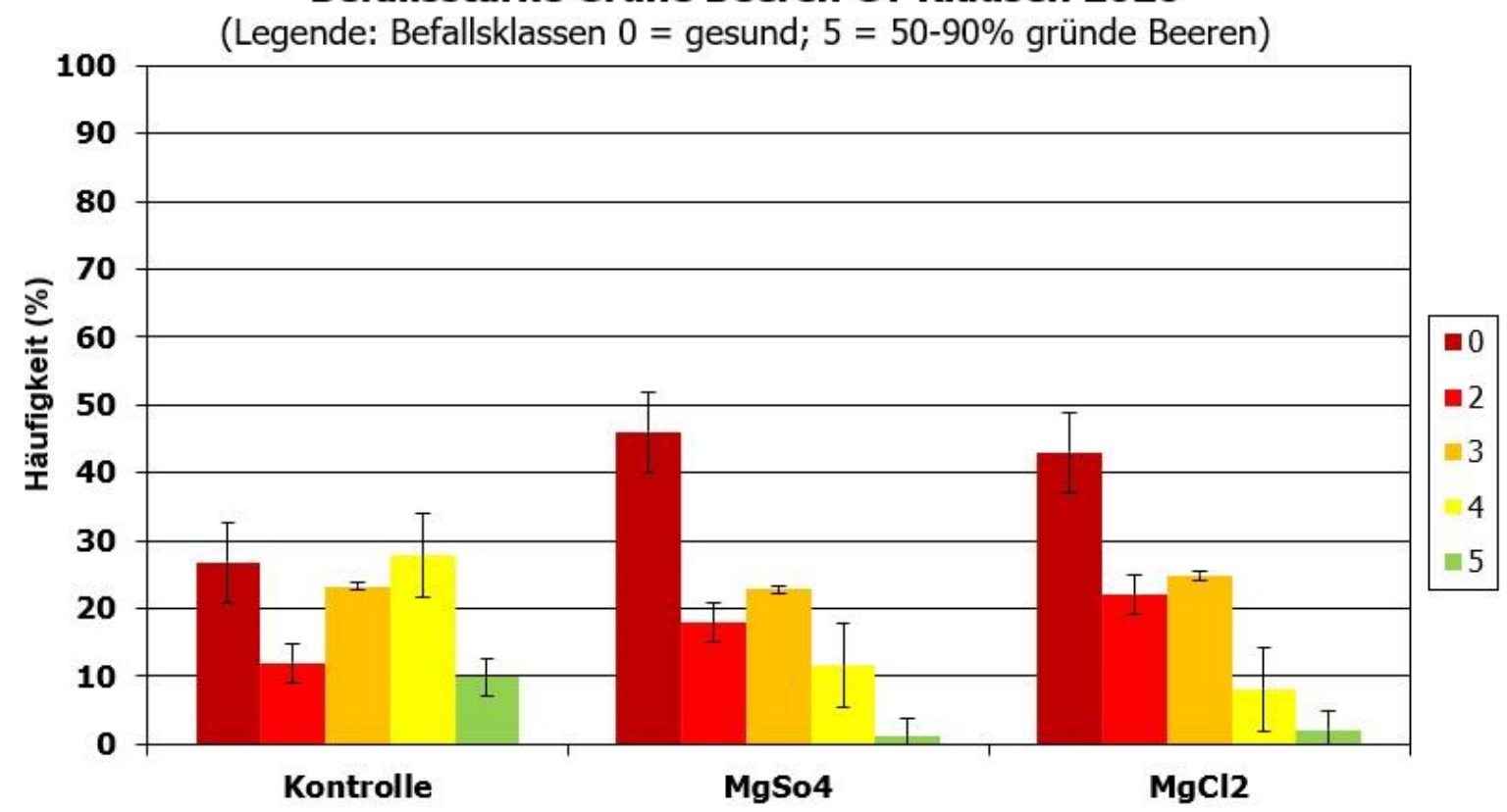

Abb. 12: Befallsstärke der "Grünen Beeren"-Auswertung am 22.09.2020 // Grape affection intensity with "green berries" among the thesis' at 22.09.2020. 


\section{DANKSAGUNG}

Die Mitarbeiter der Arbeitsgruppe Physiologie und Anbautechnik am Versuchszentrum Laimburg bedankt sich herzlich bei Philipp Höller, welcher freundlicher Weise seine Gewürztraminer-Anlage in Klausen für die Durchführung des Versuches zur Verfügung gestellt hat, für die sehr freundliche Zusammenarbeit.

\section{ZUSAMMENFASSUNG}

Die Sorte Gewürztraminer wird bekanntlich schon seit geraumer Zeit von den zwei phsyiologischen Störungen Stiellähme und Welketrauben befallen. Im Laufe der Reifephase des Jahres 2014 trat eine weitere Art der Reifestörung bei Gewürztraminer in Südtirol auf. Auffallend war, dass nicht alle Beeren den normalen Reifeprozess durchlaufen, sondern einzelne Beeren grün bleiben. In diesem Artikel werden die Ergebnisse der ersten Ursachenerforschung, sowie daraus hervorgehende erste Lösungsansätze dargestellt. Eine Behandlung mit Magnesium-Blattdünger ergab bis nun die erfolgversprechendsten Ergebnisse.

\section{RIASSUNTO}

La varietà Gewürztraminer è notoriamente affetta dalle due malattie fisiologiche disseccamento del rachide ed avvizzimento del grappolo. Durante la fase di maturazione 2014 un'altra tipologia di maturazione distorta su Gewürztraminer nell'Alto Adige. Gia in una fase precoce della maturazione dell'uva sono apparsi grappoli con singoli acini con posizione casuale sul grappolo che non hanno mirato in colore e sono rimasti verdi e aspri. Nel presente articolo sono presentati i risultati delle ricerche sulla causa della malattia, siccome le porposte di trattamento derivanti. Dei trattamenti con dei concimi fogliari di magnesio hanno mostrato dei risultati promettenti riducendo l'abbondanza e severità dell'apparenza dei sintomi.

\section{REFERENCES}

[1] Raifer B., Haas F., Cassar A. (2014). Influence of leaf canopy height on the occurrence of berry shrivel. Vitis 53 (3), 117-123, DOI: 10.5073/vitis.2014.53.117-123.

[2] Hartmair V. (1975). Beobachtungen über das Auftreten der Stiellähme in Abhängigkeit von Umweltfaktoren. Mitteilungen Klosterneuburg 25, 45-48.

[3] Versini G., Dalla Serra A., Scienza A. et al. (1990). Particolarità compositive dell'uva e del vino Traminer aromatico. Confronto fra cultivar e variazioni a livello terpenico in fermentazione e dell'invecchiamento. In: Provincia Autonoma di Bolzano - Assessorato all'agricoltura (ed.). Symposium Gewürztraminer, Simposio Traminer Aromatico, Bolzano, Italy, May 18, 1990. Provincia Autonoma di Bolzano, Bolzano, Italy, pp. 59-71, 91.
[4] Hoff R.T., Bondada B.H., Keller M. (2021). Onset and progression of the berry shrivel ripening disorder in grapes. Australian Journal of Grape and Wine Research 27 (3), 280289, DOI: 10.1111/aigw.12471.

[5] Griesser M., Eder R., Besser S. et al. (2012). Berry shrivel of grapes in Austria-Aspects of the physiological disorder with cultivar Zweigelt (Vitis vinifera L.). Scientia Horticulturae 145, 87-93, DOI: 10.1016/j.scienta.2012.07.032.

[6] Keller M. (2015). The Science of Grapewine. Accademic press, London, United Kingdom, hier pp. 306-308.
[7] Rupp D. (2012). Nährstoffversorgung und Traubenqualität. (Fachinformationen Weinbau). Staatliche Lehr- und Versuchsanstalt für Wein- und Obstbau Weinsberg, Weinsberg, Deutschland. Retrieved May 06, 2021, from https://lvwo.landwirtschaftbw.de/pb/,Lde/Startseite/Fachinformationen/Naehrstoffversorgung+und+Traubenqualitaet?LISTPAGE=670162.

[8] Reisenzein H., Berger N. (1997). Die „Zweigeltkrankheit" - eine neue Rebkrankheit? Der Winzer 63 (5), 7-9. 


\section{(c) 1 (1) $(9$}

Dieses Werk ist lizenziert unter einer Creative Commons Namensnennung-Nicht kommerziell 4.0 International Lizenz. Quest'opera è distribuita con Licenza Creative Commons Attribuzione - Non commerciale 4.0 Internazionale.

This work is licensed under a Creative Commons Attribution-NonCommercial 4.0 International License.

Für alle Abbildungen und Tabellen ohne Nennung des Urhebers gilt: (c) Versuchszentrum Laimburg. Per tutte le immagini e tabelle senza menzione dell'artefice vale: (c) Centro di Sperimentazione Laimburg. For all figures and tables without mention of the originator applies: (C) Laimburg Research Centre. 